\title{
Stromal fibroblast activation protein alpha promotes gastric cancer progression via epithelial-mesenchymal transition through Wnt/ $\beta$-catenin pathway
}

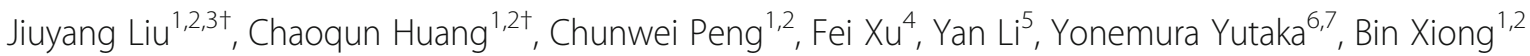
and Xiaojun Yang ${ }^{1,2^{*}}$

\begin{abstract}
Background: To investigate the influence of fibroblast activation protein alpha (FAP) derived from cancer-associated fibroblasts (CAFs), as well as potential mechanism of epithelial mesenchymal transition (EMT), on gastric cancer (GC) progression.

Methods: Correlation between CAFs-derived FAP and clinical results has been studied by using 60 GC cases. To confirm this relationship, SGC7901 cells were co-cultured with pre-established FAP-overexpressed fibroblasts in vitro and the characteristics including proliferation, migration, invasion and apoptosis abilities were detected subsequently. Meanwhile, SGC and GES1 cells cocultured with FAP-overexpressed fibroblasts were treated with cis-platinum for apoptotic analysis. The underlying EMT was detected by analyzing expression level of E-cadherin, ZO-1, N-cadherin, Vimentin, a-SMA, DKK1 and LEF-1 through western blot and immunofluorescence staining assay. Finally, the tumorpromoting ability of FAP was investigated by utlizing a xenograft gastric cancer nude mouse model.

Results: It show that FAP has a high-risk correlation with the malignant level of clinical outcomes in GC patients. FAP promotes the ability of proliferation, migration, invasion, apoptosis-inhibition of SGC7901 cells and induces apoptosis of GES1 cells in vitro. The mechanism study shows that epithelial markers have been down-regulated and mesenchymal markers and Wnt/ $\beta$-catenin signal pathway related proteins have been up-regulated. Animal assay suggests that tumor burden has been enhanced by FAP significantly in vivo.
\end{abstract}

Conclusions: Stromal FAP could be a potential prognostic biomarker in GC by promoting cancer progression via EMT through Wnt/ $\beta$-catenin signal pathway.

Keywords: Gastric cancer, Peritoneal metastasis, Fibroblast activation protein alpha, Epithelial-mesenchymal transition

\section{Background}

Gastric cancer (GC) remains the fourth most common cancer and the fifth leading cause of cancer-related mortality worldwide $[1,2]$. The postoperative invasion and metastasis have long been the lethal causes of death and great challenges for GC patients even after multimodality

\footnotetext{
* Correspondence: totti800105@163.com

${ }^{+}$Liu Jiuyang and Huang Chaoqun contributed equally to this work.

'Department of Gastrointestinal Surgery, Zhongnan Hospital of Wuhan

University, No. 169 Donghu Road, Wuchang District, Wuhan, China

${ }^{2}$ Hubei Key Laboratory of Tumor Biological Behaviors \& Hubei Cancer Clinical

Study Center, Wuhan 430071, China

Full list of author information is available at the end of the article
}

clinical treatments [3]. And almost $60 \%$ of all causes of GC death is due to peritoneal carcinomatosis (PC) [4]. According to recent new insights, $\mathrm{PC}$ was regarded as a regional tumor progression majorly occurred in abdomen pelvic cavities $[5,6]$.

The underlying mechanisms of GC PC has been a worldwide research hotspot, and more efforts were focused on the dynamic and complex PC progression. Momentum evidence has indicated that tumor microenvironment (TME) plays a crucial role in cancer progression [7, 8]. The co-evolution of cancer cells and stromal functional cells or molecules constitutes significant hallmarks of cancer [9].

(c) The Author(s). 2018 Open Access This article is distributed under the terms of the Creative Commons Attribution 4.0 International License (http://creativecommons.org/licenses/by/4.0/), which permits unrestricted use, distribution, and reproduction in any medium, provided you give appropriate credit to the original author(s) and the source, provide a link to the Creative Commons license, and indicate if changes were made. The Creative Commons Public Domain Dedication waiver (http://creativecommons.org/publicdomain/zero/1.0/) applies to the data made available in this article, unless otherwise stated. 
Cancer associated fibroblasts (CAFs) act as key orchestrators in TME by directly protecting cancer cells from host immune attacks, and promoting cancer progression by complex mechanisms, for instance epithelial-mesenchymal transition (EMT) [10, 11]. Whether EMT could partly explain the cross talk between GC cells and stromal CAFs required further studies [12].

Fibroblast activation protein alpha (FAP), a homodimeric integral membrane gelatinase of the serine protease family, is selectively expressed by CAFs in stromal compartment $[13,14]$. FAP could exerte profound influence on clinical outcomes of several human malignancies. For instance, FAP overexpression correlated with suppressed lymphocyte-dependent immune reactions and poor survival of non-small cell lung cancer and pancreatic adenocarcinoma $[15,16]$. However, stromal FAP derived from CAFs in GC remained to be confirmed, as well as the regulatory mechanisms [17].

In this study, we have conducted experiments in vitro and in vivo to further characterize the biological processes associated with stromal FAP overexpression in GC. Based on the pre-established FAP-overexpressed fibroblasts $\left(\mathrm{HELF}^{\mathrm{FAP}}\right)$, the proliferation, invasion, migration, as well as anti-apoptosis abilities of SGC7901 cells in co-cultured model were investigated. Moreover, correlations between FAP and $\mathrm{Wnt} / \beta$-catenin pathway was also detected to ascertain the potential role of EMT during GC progression. Taken together, we described the tumor promoting functions of stromal FAP, which might account for GC progression.

\section{Materials and methods}

\section{Patients and follow-up}

There were $60 \mathrm{GC}$ cases included in this study, all of which have received radical operation at the Department of Gastrointestinal Surgery, Zhongnan Hospital of Wuhan University (Wuhan, China) from February 2009 to April 2011. Major clinicopathological characteristics including age, gender, tumor diameter, and TNM stages were collected. In addition, the information of follow up was available. TNM stages were determined according to the UICC/AJCC 7th TNM staging system of GC. The primary endpoint for this study was overall survival (OS), which was defined as the interval from the date of surgery to GC related death. Written informed consent was obtained from the patients with the study protocol approved by the ethics committee of Zhongnan Hospital of Wuhan University. The study was undertaken in accordance with the ethical standards of the World Medical Association Declaration of Helsinki.

\section{Immunohistochemistry staining}

Routine IHC method was performed for the staining of FAP. The primary antibody was rabbit anti-human monoclonal antibody against FAP (ab227703, Abcam, UK, dilution 1/200), with corresponding horseradish peroxidase (HRP) conjugated secondary antibody (ab6721, Abcam, UK, dilution 1/200). The FAP positive CAFs were indicated by both morphological features and the IHC reaction results. The reaction products were visualized with diaminobenzidine (DAB, DAKO, Denmark). Then the slides were evaluated by two senior pathologists, who were blinded to the patients' clinical features and outcomes. A consensus was achieved using a multi-headed microscope in case of discrepancy. In brief, at least 4 standard-compliant vision fields of FAP expression (magnification, $\times 200$ ) per patient was considered to be adequate, with no focus on hotspots. The digital images were captured under Olympus BX51 fluorescence microscope equipped with Olympus DP72 camera (Olympus Optical Co., Ltd., Tokyo, Japan). Identical settings were used for every photograph, so as to minimize the selection bias.

\section{Cell culture}

The SGC7901 cell line (human gastric cancer cell lines), GES1 cell line (normal mucosal epithelium cells), and HELF cell line (human embryonic lung fibroblasts; Cat NO.: CL-0281) were cultured in Dulbecco's modified Eagle's medium (DMEM) supplemented with 10\% Fetal Bovine Serum (FBS), $100 \mathrm{IU} / \mathrm{ml}$ penicillin and $100 \mathrm{mg} /$ $\mathrm{ml}$ streptomycin in a humidified atmosphere with $5 \%$ $\mathrm{CO}_{2}$ at $37^{\circ} \mathrm{C}$.

\section{Construction of HELF ${ }^{\text {FAP }}$ cells with overexpression of FAP}

The lentivirus FAP-copGFP $\left(1 \times 10^{8} \mathrm{TU} / \mathrm{ml}\right)$ and a negative control (NC) were purchased from GenePharma (Shanghai, China). HELF cells seeded in six-well plates were transfected with control or lentivirus FAP-copGFP according to the manufacturer's instructions. The multiplicity of infection (MOI) in this study was 50:1. Then puromycin was used to establish the stable transfected HELF cell line $\left(\operatorname{HELF}^{\mathrm{FAP}}\right)$. SGC7901 co-cultured with HELF $^{\mathrm{FAP}}$ and $\mathrm{HELF}^{\mathrm{NC}}$ cells were used for further experiments.

\section{CCK8 assay}

Cholecystokinin-8 (CCK-8) assay (Dojindo, Japan) was performed to detect the cell viability and cell growth. Briefly, 6000 viable gastric cancer cells were seeded in 96-well plates. After specific treatment, each well was mixed with $10 \mu \mathrm{L}$ CCK- 8 and incubated for additional $1 \mathrm{~h}$. The OD values were detected at an absorbance of $450 \mathrm{~nm}$. 


\section{Colony formation assay}

A colony formation assay was used to detect cells survival. For clonogenicity analysis, 1000 viable co-cultured SGC7901 cells were placed in six-well plates. Culture medium was changed every two days. After two weeks of incubation, colonies were fixed with $4 \%$ paraformaldehyde and stained with crystal violet. The cells were photographed and the numbers of colonies were scored.

\section{Wound healing assay}

SGC7901 cells seeded in 6-well plates were scratched, washed with PBS supplemented with 1\% FBS and treated as indicated. The cells were photographed by phase contrast microscope at $24 \mathrm{~h}$ in several pre-marked spots. Then the mean distance between both edges of cell free area was calculated.

\section{Transwell migration and invasion assays}

The polycarbonate membrane in the transwell chambers were precoated with Matrigel with 1:40 dilution (Corning, USA) in $37{ }^{\circ} \mathrm{C}$ and air dried. There were 15,000 cells seeded and adhered in each chamber. After $24 \mathrm{~h}$, the cells were fixed with $4 \%$ paraformaldehyde (PFA) and stained by $0.1 \%$ crystal violet, the number of migrated cells were counted and statistically analyzed. For migration assay, no Matrix gel was required.

\section{Flow cytometry}

SGC7901 cells were placed in 12-well plates overnight, and then treated with compounds according to the manufacturer. Cells were then harvested, washed twice with pre-cold PBS, and evaluated for apoptosis by double staining with FITC-conjugated annexin V and propidium iodide (PI) (MultiSciences, Hangzhou, China) for $30 \mathrm{~min}$ in the dark. To assess the cell cycle, harvested cells were labeled with PI $(5 \mathrm{mg} / \mathrm{ml})$ in the presence of binding buffer (MultiSciences, Hangzhou, China) in darkness for $30 \mathrm{~min}$.

\section{Real-time RT-PCR}

Total RNA was extracted using RNA simple Total RNA kit (TIANGEN, Beijing, China). cDNA was generated with a first-strand cDNA synthesis Kit (Thermo, Waltham, MA) using the protocol recommended by the manufacturer.

The one-step real-time quantitative PCR were carried out in a $20 \mu \mathrm{l}$ reaction mixture containing $10 \mu \mathrm{l} 2 \times$ SYBR Premix EX Taq II (Takara, Tokyo, Japan), $0.4 \mu \mathrm{M}$ primers, and $1 \mu \mathrm{l}$ of template cDNA. The primers were listed in Additional file 1: Table S1. All real-time RT-PCRs were performed at CFX96 real-time PCR detection system (Bio-Rad, Hercules, CA). Fluorescence was measured at the end of the annealing period of each cycle to monitor amplification. Glyceraldehyde-3-phosphate dehydrogenase $(\mathrm{GAPDH})$ was used as internal reference.

\section{Western blotting}

Cells were washed with cold PBS twice and prepared in RIPA lysis buffer, and western blot analysis was performed as described previously [18]. Specific primary antibodies used were the following: DKK1 (ab61275, Abcam); LEF1 (ab217378, Abcam); ZO-1 (61-7300, Thermo); Vimentin (ab92547, Abcam); N-cadherin (ab76011, Abcam); E-cadherin (ab1416, Abcam). Anti-GAPDH was purchased from Aspen (Wuhan, China). After incubating with a fluorescein-conjugated secondary antibody (Li-Cor, Lincoln, NE, USA), the membranes were analyzed using an Odyssey fluorescence scanner (Li-Cor, Lincoln, NE, USA).

\section{Immunofluorescence staining (IF)}

SGC7901 cells were seeded on $24 \mathrm{~mm}$ coverslips, fixed with $4 \%$ PFA for $30 \mathrm{~min}$, treated by $0.1 \%$ Triton X-100 and blocked in 5\% BSA for $1 \mathrm{~h}$ at room temperature. Sequentially the fixed cells were incubated with primary antibody at $4{ }^{\circ} \mathrm{C}$ overnight (E-cadherin, ab1416, Abcam, dilution 1/50; $\alpha$-SMA, ab32575, Abcam, dilution 1/300), washed with PBS and incubated with Cy3-labelled or FITC-labelled secondary antibody for $1 \mathrm{~h}$ at room temperature. The nuclei were labelled with DAPI $(2 \mathrm{mg} /$ $\mathrm{ml}$ ), and the immunofluorescence staining was analyzed using a fluorescence microscope (Olympus BX5, Olympus Optical Co., Ltd., Tokyo, Japan).

\section{In vivo xenograft assay}

Six-week-old female BALB/cA nu/nu mice were purchased from Vital River Laboratory Animal Technology Company (Beijing, China) and maintained in an Animal Biosafety Level 3 Laboratory at the Animal Experimental Center of Wuhan University. All animal experiments were performed according to the Wuhan University Animal Care Facility and National Institutes of Health guidelines. Approximately $3 \times 10^{6}$ SGC7901 cells and $1 \times 10^{6}$ HELF $^{\text {FAP }}$ cells (Group I, $n=5$ ), $3 \times 10^{6}$ SGC7901 cells and $1 \times 10^{6}$ HELF $^{\mathrm{NC}}$ cells (Group II, $\mathrm{n}=5$ ) were harvested and suspended in $200 \mathrm{ml}$ of PBS and Matrigel (BD Bio-science, USA) (1:1) and injected subcutaneously into the right flank of each mouse. The size of subcutaneous tumors was recorded every two days. Five weeks later, mice were sacrificed, and the tumors were removed. The weight of tumors was recorded and statistically analyzed. The xenograft tumor slides were incubated with the following primary antibodies: anti-CD31 was purchased from ABclonal (Boston, USA) 
Table 1 The relationship between stromal FAP and pathological characteristics in patients with gastric cancer

\begin{tabular}{llll}
\hline Variables & No. (\%) & FAP positive CAFs & $P^{*}$ \\
\hline Gender & & & 0.309 \\
$\quad$ Male & $35(62.5 \%)$ & $34.8 \pm 12.6$ & \\
$\quad$ Female & $25(37.5 \%)$ & $30.7 \pm 11.5$ & \\
Age (Means \pm SD, yrs) & & & 0.254 \\
$\quad<60$ & $32(55.0 \%)$ & $38.9 \pm 10.1$ & \\
$\quad \geq 60$ & $28(45.0 \%)$ & $35.7 \pm 13.2$ & \\
Tumor diameter & & & $\mathbf{0 . 0 2 4}$ \\
$\quad<5$ cm & $34(60.0 \%)$ & $28.2 \pm 15.2$ & \\
$\quad \geq 5$ cm & $26(40.0 \%)$ & $42.8 \pm 20.4$ & \\
Differentiation degrees & & & $\mathbf{0 . 0 0 2}$ \\
$\quad$ Poorly-differentiated & $28(45.0 \%)$ & $45.4 \pm 13.0$ & \\
$\quad$ Moderately-differentiated & $17(30.0 \%)$ & $35.6 \pm 15.5$ & \\
$\quad$ Well-differentiated & $15(25.0 \%)$ & $16.3 \pm 8.6$ & $\mathbf{0 . 0 0 1}$ \\
TNM stage & & & \\
Stage I/II & $25(42.5 \%)$ & $24.5 \pm 6.4$ & \\
Stage III/IV & $35(57.5 \%)$ & $57.1 \pm 20.1$ & \\
\hline
\end{tabular}

* $P$-value in bold indicates the difference was statically significant and anti-Ki67 from Cell Signaling Technology (Boston, USA). Anti-rabbit or anti-mouse peroxidaseconjugated secondary antibody (ABclonal, Boston, USA) and diaminobenzidine colorimetric reagent solution (Dako, Carpinteria, CA) were used. The staining processes were performed according to standard methods.

\section{Statistical analysis}

All experiments were performed at least three times. Data are presented as the mean $\pm \mathrm{SD}$. All statistical analyses were performed using GraphPad Prism 6.0 (GraphPad, San Diego, CA). One-way ANOVA and Student's t-test were applied to determine statistical significance. A value of two-sided $P<0.05$ was considered statistically significant.

\section{Results}

The clinical significance of stromal FAP in GC

A total of 60 patients were included in this study, detailed information about patients' demographics, clinicopathological characteristics was shown in Table 1 . There were 4 groups including I $(n=12)$, II $(n=13)$, III $(n=$ $27)$, and IV $(n=8)$. FAP was mainly expressed in cancer cells or CAFs (Fig. 1a, b). The positive ratio of FAP was $91.7 \%$ in GC tissues $(n=55)$. The FAP positive CAFs in GC tissues $(32.80 \pm 19.3)$ was much higher than that in
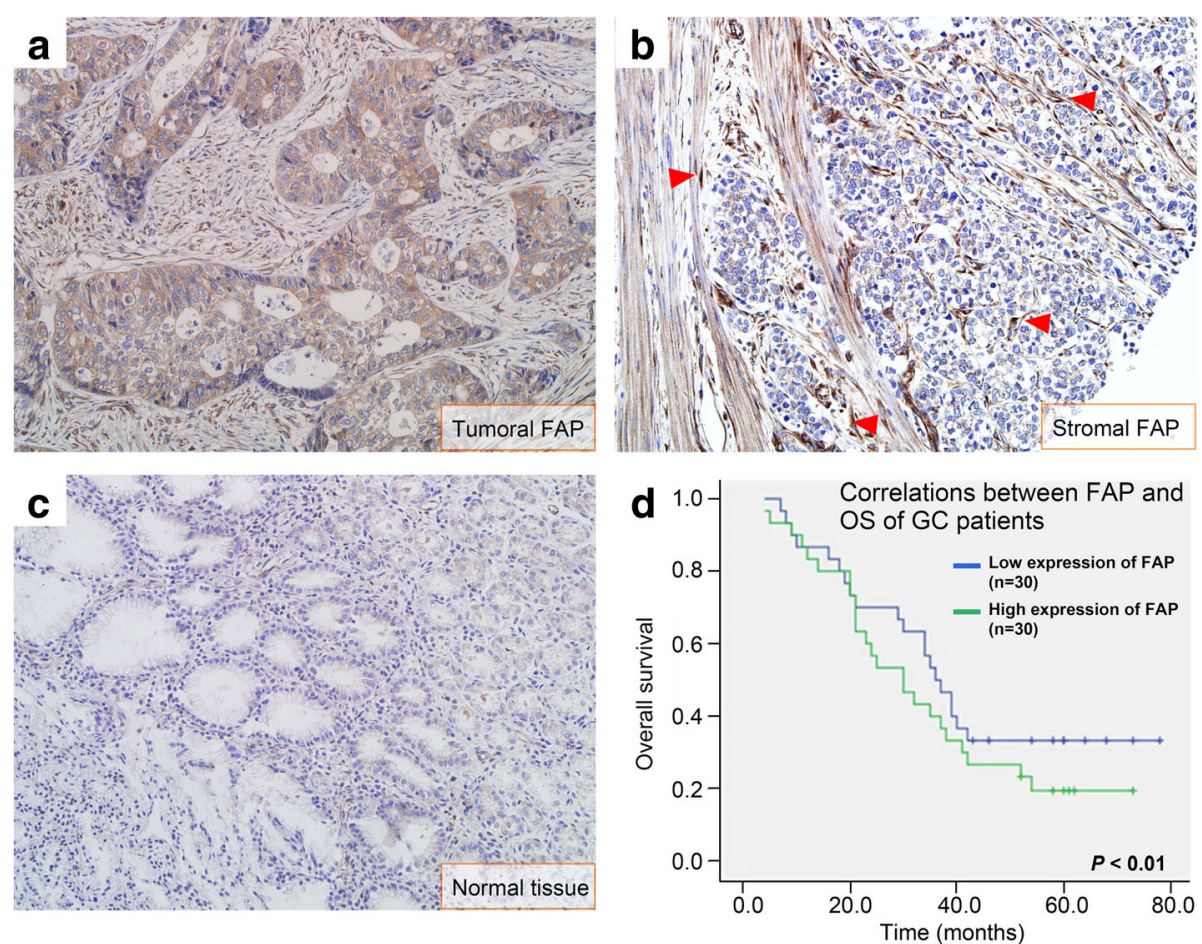

Fig. 1 Correlations between FAP and OS of GC patients. FAP was expressed both in GC cells (a) and stromal CAFs (b, shown by red arrowheads). c FAP was not expressed in normal tissues. $\mathbf{d}$ The median OS of GC cases with high expression of FAP (30.2 months) was shorter than that with low expression of FAP (37.8 months), the difference was statically different $(P<0.01)$ 
peritumoral tissues $(0.41 \pm 0.21)$, the difference was statically different $(P<0.01)$.

FAP expression correlated with the tumor diameter $(P$ $=0.024)$, tumor differentiation degrees $(P=0.002)$, and TNM stage $(P=0.001)$, but not correlated with age and gender $(P>0.05$ for all). According to the median value of FAP positive CAFs, GC cases were divided into high expression of FAP group $(n=30)$ and low expression of FAP group $(n=30)$. The median OS of GC cases with high expression of FAP (30.2 months) was shorter than that with low expression of FAP (37.8 months), the difference was statically different $(P<0.01$, Fig. 1 c).

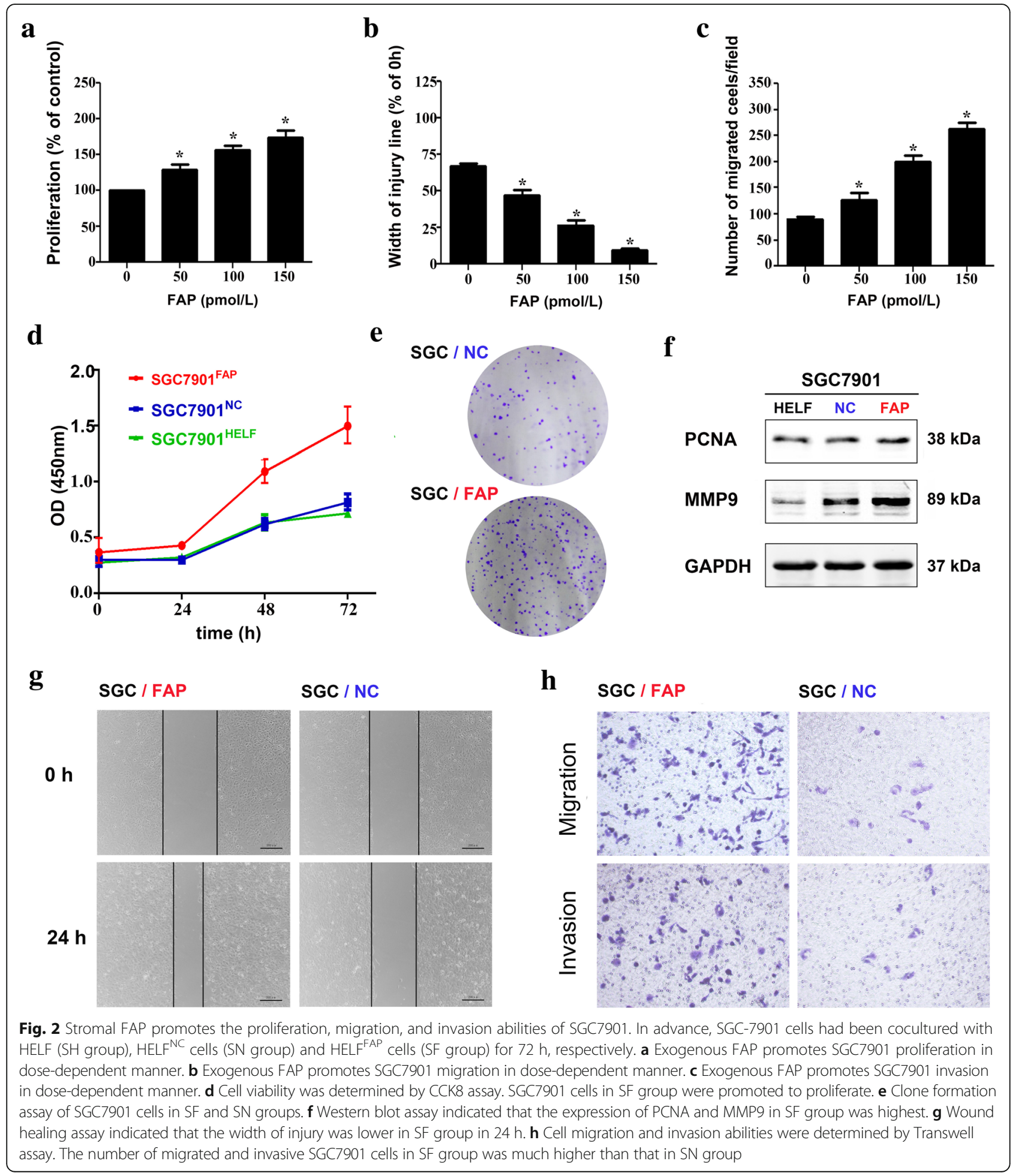


The construction of HELF ${ }^{\mathrm{FAP}}$ cells with overexpression of FAP

The infection efficiency of FAP-copGFP was 100\% at $72 \mathrm{~h}$ after infection and puromycin-based screening, which was indicated by green fluorescence. FAP expression was significantly elevated in HELF $^{\mathrm{FAP}}$ cells by nearly sixtyfold than $\mathrm{HELF}^{\mathrm{NC}}$ cells. The IF results also indicate significantly higher FAP protein expression within $\operatorname{HELF}^{\mathrm{FAP}}$ cells (Additional file 2: Figure S1). Therefore, HELF $^{\mathrm{FAP}}$ cells with overexpression of FAP were constructed for further studies, including cell proliferation, migration, invasion, as well as apoptosis.
Stromal FAP promotes the proliferation, migration, and invasion abilities of SGC7901

The proliferation and migration abilities of SGC7901 were significantly elevated by exogenous FAP in dose-dependent manner, as shown in Fig. 2a-c. The co-culture system went a further step to confirm this phenomenon. After co-cultured with HELF ${ }^{\mathrm{FAP}}, \mathrm{HELF}^{\mathrm{NC}}$ and HELF cells for $72 \mathrm{~h}$, SGC7901 cells were harvested for CCK8 assays. The OD (450) value was recorded every $24 \mathrm{~h}$ to draw the proliferation curve, which indicated that the OD value of SGC7901 ${ }^{\mathrm{FAP}}$ was much higher (Fig. 2d). The number of SGC7901 ${ }^{\text {FAP }}$ cells colony was also much higher than that of SGC7901 ${ }^{\mathrm{NC}}$

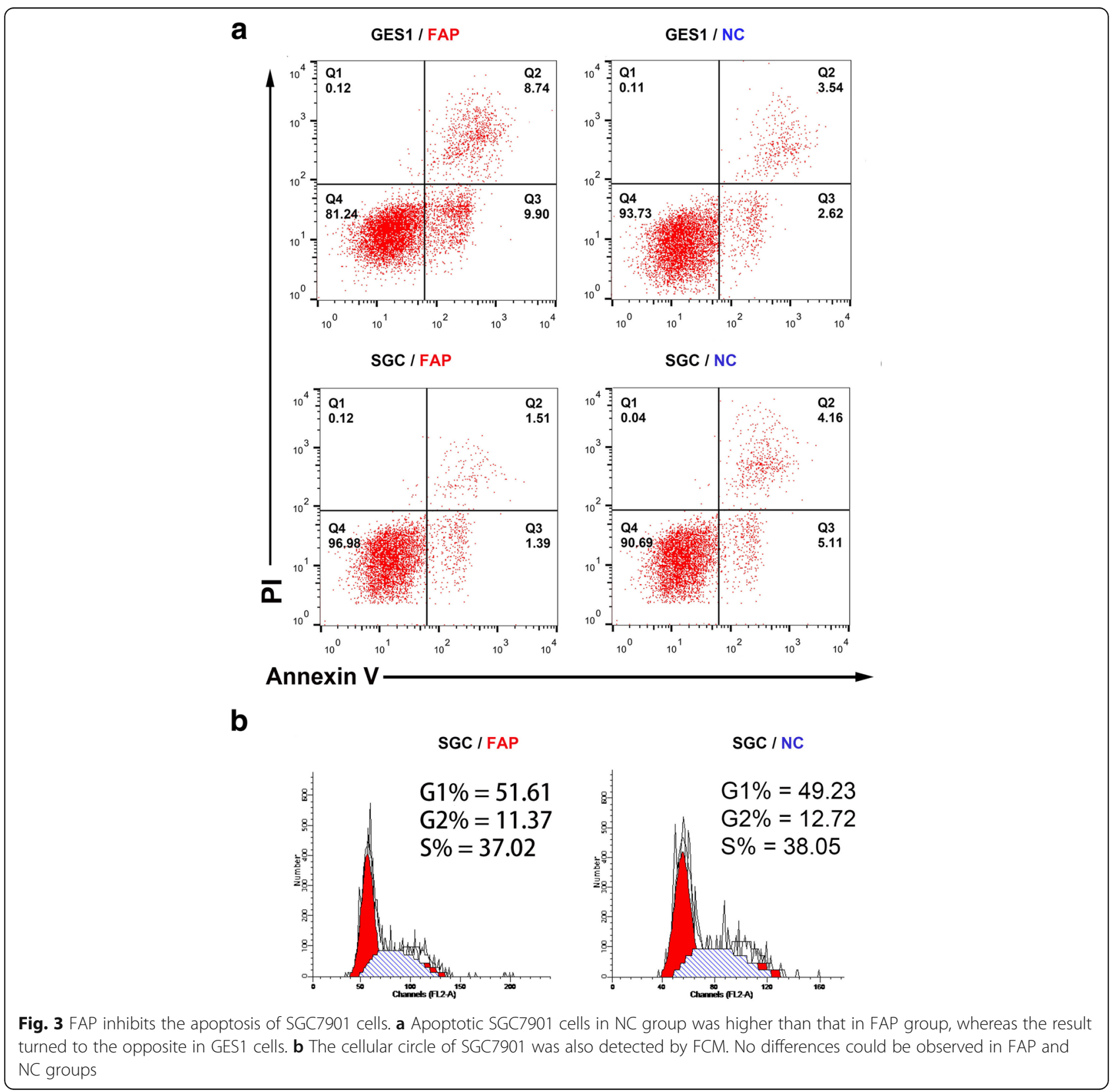


(Fig. 2e). Western blot assay indicated that the expression of PCNA and MMP9 protein in SGC7901 ${ }^{\mathrm{FAP}}$ were highest (Fig. 2f). The width of injury was lower in SGC7901 ${ }^{\mathrm{FAP}}$ cells in $24 \mathrm{~h}$ by wound healing assay (Fig. 2g). Cell migration and invasion abilities were determined by Transwell assay. The number of migrated and invasive SGC7901 cells in FAP group was much higher than that in NC group (Fig. 2h).

\section{Stromal FAP inhibits the apoptosis of SGC7901 cells}

The cocultured GES1 and SGC7901 cells were treated with cis-platinum meanwhile, then the apoptosis effect was detected by flow cytometry (FCM). Apoptotic SGC7901 cells in NC group was higher than that in FAP group, whereas the result turned to the opposite in GES1 cells (Fig. 3a). The cellular circle of SGC7901 was also detected by FCM to evaluate the potential reasons of apoptosis. However, no differences could be observed between FAP and NC groups (Fig. 3b). Then we hypothesized the potential correlation between FAP and Caspase family considering the apoptosis effect, whereas Western blot assay indicated that no significant differences regarding the expression of Caspase3, Caspase 9, Bax and Bcl-2 between FAP and $\mathrm{NC}$ groups (Additional file 3: Figure S2).

\section{Stromal FAP promotes EMT of SGC7901 through Wnt/ $\beta$-catenin pathway}

Exogenous FAP promotes EMT in dose-dependent manner. The expression of E-cadherin and ZO-1 were reduced, while that of $\mathrm{N}$-cadherin and Vimentin were increased by qRT-PCR assay (Fig. 4a), and Western blotting assay (Fig. 4b). In addition, the DKK1 and LEF-1 protein, which could be participated in Wnt/ $\beta$-catenin a

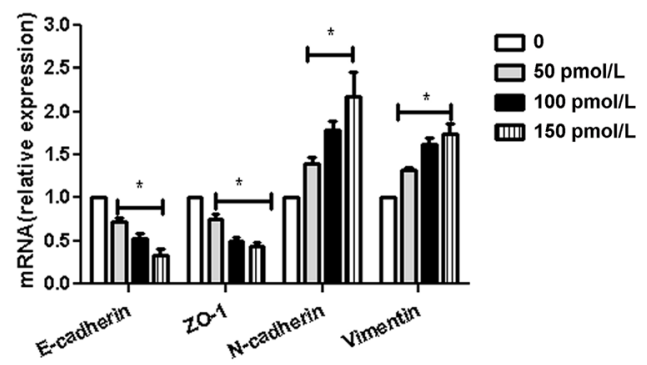

C
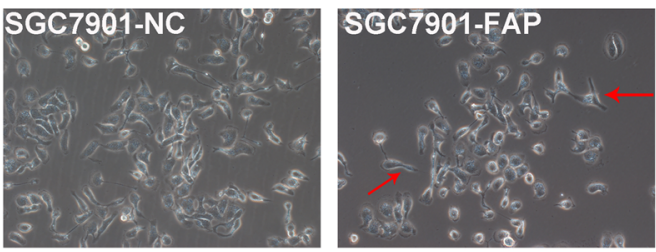

d

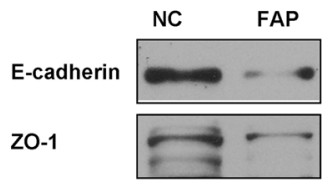

Vimentin

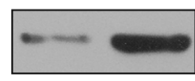

$\mathrm{N}$-cadherin
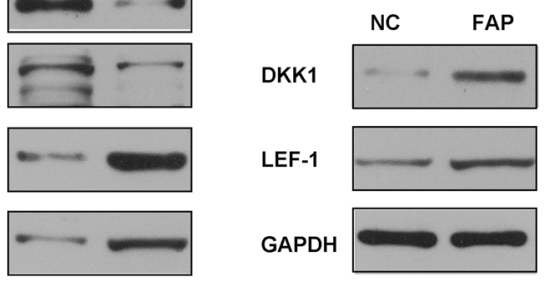

b

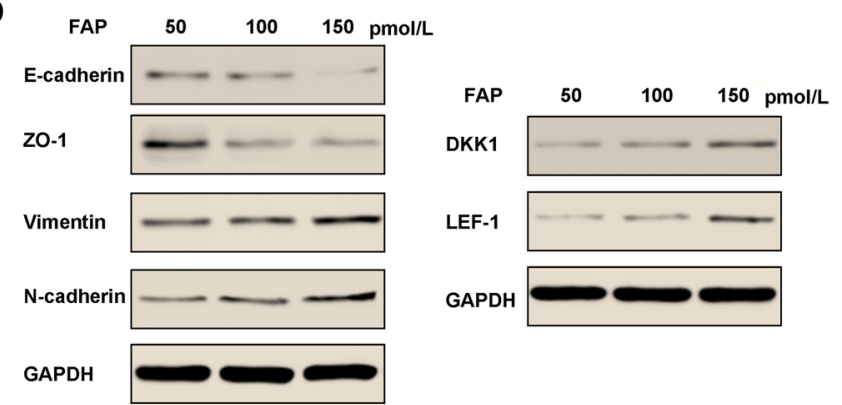

e
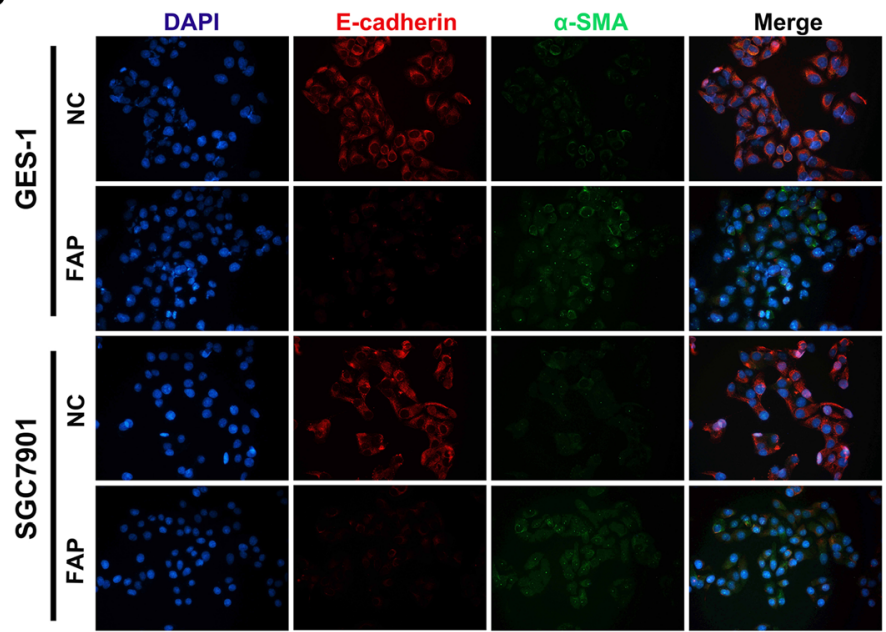

Fig. 4 FAP promotes EMT of GC cells through Wnt/ $\beta$-catenin pathway. Exogenous FAP promotes SGC EMT in dose-dependent manner. The expression of E-cadherin and ZO-1 were reduced, while that of N-cadherin and Vimentin were increased by qRT-PCR assay (a), and Western blotting assay (b). In addition, the DKK1 and LEF-1 protein, which could be participated in Wnt/ 3 -catenin pathway, were also increased with more exogenous FAP. The result also accompanied in SGC cells co-cultured in SN and SF groups. c The morphology of SGC cells in SF group tended to be fibroblast-like, long fusiform, which was indicated by red arrows. $\mathbf{d}$ The expression of E-cadherin and ZO-1 were reduced, while that of Ncadherin and Vimentin were increased in SGC cells of SF group by Western blotting assay. Similarly, the DKK1 and LEF-1 protein were also increased. e The expression of E-cadherin was reduced, while that of a-SMA was increased both in GES1 cells (gastric normal cells) and SGC cells of SF group by the immunofluorescence staining 
pathway, were also increased with more exogenous FAP. The result also accompanied in SGC7901 cells co-cultured in NC and FAP groups.

The morphology of SGC7901 cells in FAP group tended to be fibroblast-like, long fusiform, which was indicated by red arrows in Fig. 4c. The expression of E-cadherin and ZO-1 were reduced, while that of $\mathrm{N}$-cadherin and Vimentin were increased in SGC cells of SF group by Western blotting assay. Similarly, the DKK1 and LEF-1 protein were also increased (Fig. 4d). The expression of E-cadherin was reduced, while that of $\alpha$-SMA was increased both in GES1 cells and SGC7901 cells of FAP group by the IF staining (Fig. 4e).

\section{Stromal FAP promotes GC progression in a xenograft gastric cancer nude mouse model}

To investigate the in vivo effects of stromal FAP, we examined the tumor promoting effect of FAP in a xenograft gastric cancer nude mouse model. SGC7901 cells $\left(3 \times 10^{6}\right)$ were implanted subcutaneously in the right flank of nude mice, accompanied with $\operatorname{HELF}^{\text {FAP }}\left(1 \times 10^{6}\right)$ $(n=5)$ and $\operatorname{HELF}^{\mathrm{NC}}\left(1 \times 10^{6}\right)(\mathrm{n}=5)$ cells, respectively. The combination of SGC7901 and HELF ${ }^{\mathrm{FAP}}$ was much more effective in elevating tumor burden (Fig. 5a). The tumor volume and weight in the NC group were significantly lower than FAP group (Fig. 5b, c). Ki67 and CD31were examined by immunohistochemistry in the tumor sections. Both Ki67 and CD31 expression were elevated in FAP group. Taken together, stromal FAP promotes GC progression in a xenograft gastric cancer nude mouse model.

\section{Discussion}

In this study, stromal FAP levels correlated with adverse clinic-pathological characteristics in GC, including larger tumor diameter, poorly tumor differentiation degrees, and advanced TNM stage. Therefore, FAP overexpression might contribute to cancer progression. Similar results could be summarized in colorectal cancer [19], pancreatic adenocarcinoma [20] and esophageal malignancies [21]. Unlike previous studies, our work provided a new insight into stromal FAP derived from CAFs in microenvironment [14]. The number of FAP positive CAFs were used to stratify GC patients into low- and high-risk groups. Consequently, the median OS of high-risk group was shorter. Therefore, stromal FAP might be closely related to GC progression and a potential prognostic biomarker.

Further biochemical and animal studies were conducted to ascertain the role of FAP as a causative and mechanistic biomarker. Although previous studies illustrated that FAP could promote cancer cells proliferation and invasion in various malignancies, for instance HO-8910 PM ovarian cancer cells [22], the TME-derived
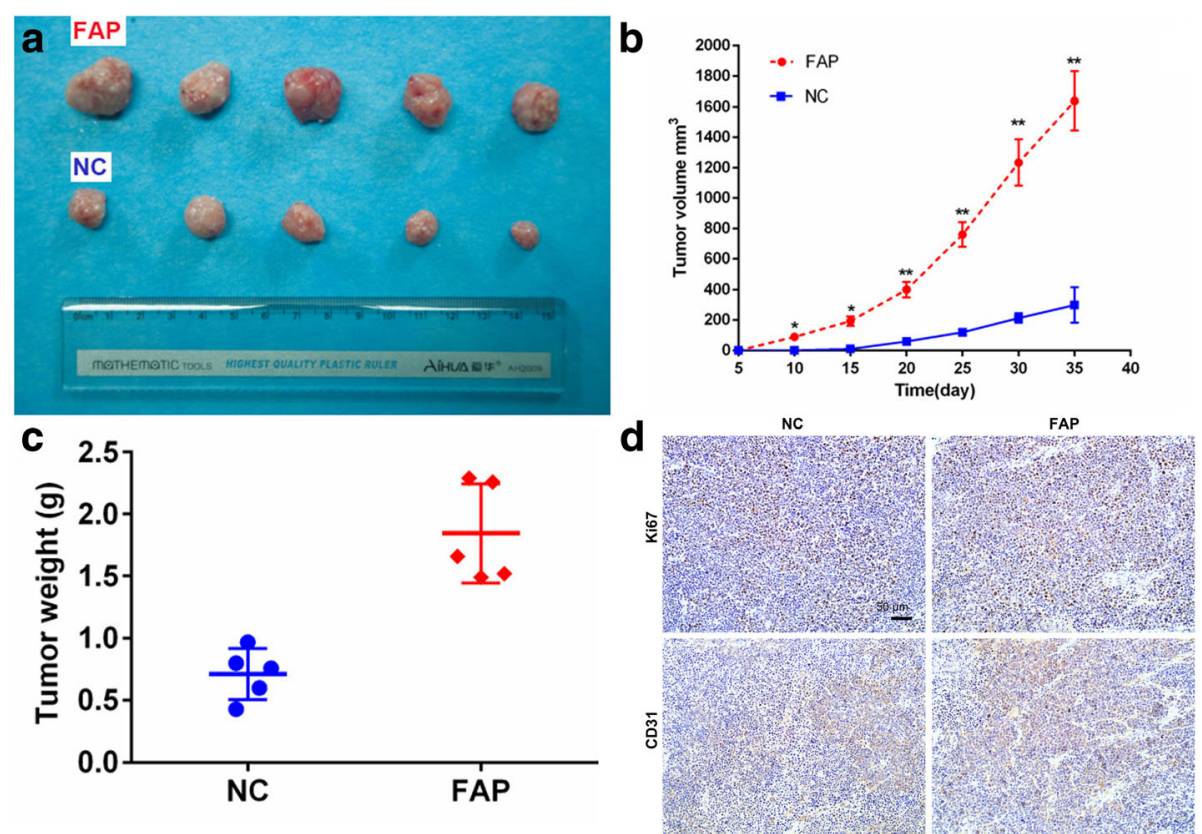

Fig. 5 FAP promotes GC progression in a xenograft gastric cancer nude mouse model. a The combination of SGC7901 and HELFFAP was much more effective in elevating tumor burden. The tumor volume $(\mathbf{b})$ and weight $(\mathbf{c})$ in the NC group were significantly lower than FAP group. $\mathbf{d}$ Representative immunohistochemical analysis of CD31, Ki67 (200x magnifications, Scale bar 50 mm) 
causations were ignored. Momentum evidence had confirmed the predominant function of TME during cancer invasion and metastasis [23-25]. In fact, the tumor initiation and growth were partially depended on stromal CAFs [11, 26]. According to TME theory, a co-culture model and a xenograft nude mouse model were used to mimic the cross talk between CAFs and GC cells. Herein, the exogenous FAP and HELF $^{\mathrm{FAP}}$ cells were found to promote the proliferation, migration and invasion abilities of GC cells in vitro by a series of functional assays. Therefore, it went a step further to detect tumor promoting functions of stromal FAP.

Except for sustaining proliferative abilities, resisting cell death and apoptosis was also the hallmarks of cancer [27]. Herein, stromal FAP inhibited the GC apoptosis, but induced normal mucosa epithelium apoptosis. Hence stromal FAP might be tumorigenic by destroying gastric epithelial cells and sustaining GC malignancies. Then we could hypothesize that, like other stromal components [28], CAFs were remodeled to support GC progression. As known, the main effect of apoptosis was mediated by Caspase-3 [29] and Caspase-9 [30] activation. The pro-apoptosis protein Bax might also be involved in by releasing cytochrome $\mathrm{c}$ from mitochondria and caspasedependent pathway [31]. In this study, no similar phenomena could be found, thereby making it necessary to further explore underlying mechanisms.

Accumulating evidence indicated that EMT was a complex and dynamic process utilized by cancer cells during invasion and metastasis [32]. Once EMT occurred, cells lose the cell polarity and cell-cell contact, and gain mesenchymal properties, for instance increased motility [33]. The inducers of EMT can downregulate $\mathrm{E}$-cadherin and upregulate $\mathrm{N}$-cadherin and vimentin through modulating EMT-related signaling pathways, for instance $\mathrm{WNT} / \beta$-catenin [34]. Dkk1, an antagonist of $\mathrm{Wnt} / \beta$-catenin signaling, partially reverses the expression of EMT-associated proteins [35], and inversely correlated with cells apoptosis [36]. Herein, we reported corresponding results of E-cadherin, ZO-1, N-cadherin, vimentin, DKK1, and LEF-1. As a result, the above discussed functional roles of stromal FAP could be induced by EMT through $W n t / \beta$-catenin signaling.

\section{Conclusion}

In summary, we went a step further to characterize the biological processes and potential mechanisms associated with stromal FAP overexpression in GC. Stromal FAP derived from CAFs could promote GC progression via EMT mechanism through $\mathrm{Wnt} / \beta$-catenin pathway.

\section{Additional files}

Additional file 1: Table S1. Primers sequences in this study. (DOCX 15 $\mathrm{kb})$

Additional file 2: Figure S1. The construction and identification of HELF FAP cells. (a) HELF FAP cells in the bright field and the fluorescence field. The infection efficiency of FAP-copGFP was $100 \%$ at $72 \mathrm{~h}$ after infection. (b) The expression of FAP in HELFFAP cells was significantly elevated in HELF ${ }^{\text {FAP }}$ cells by nearly sixtyfold through qRT-PCR assay, the difference was statically different $(P<0.001)$. (c) The immunofluorescence staining of FAP protein in both HELF ${ }^{N C}$ and HELF ${ }^{F A P}$ cells. FAP was overexpressed in HELF ${ }^{\text {FAP }}$ cells. (JPG 6016 kb)

Additional file 3: Figure S2. Western blot assay indicated that no significant differences were found regarding the expression of caspase3, caspase 9, Bax and BCl-2 in SGC7901 cells between FAP and NC groups. (JPG $217 \mathrm{~kb}$ )

\section{Abbreviations}

CAFs: cancer-associated fibroblasts; EMT: epithelial-mesenchymal transition; FAP: fibroblast activation protein; GC: gastric cancer; IHC: immunohistochemistry; OS: overall survival; PC: peritoneal carcinomatosis; TME: tumor microenvironment

\section{Acknowledgements}

Not applicable.

Funding

This work was supported by Science Fund of the National Natural Science Foundation of China (No. 81502113).

Availability of data and materials

All the data is contained in the manuscript.

\section{Authors' contributions}

$J Y L, C Q H, C W P$ and XJY conceived of the study and participated in its design and coordinated and helped to draft the manuscript. JYL, CQH, and XJY performed the experiments. XJY and YL participated in the design of the study and performed the statistical analysis. JYL, CQH, CWP and XJY wrote the paper. All authors read and approved the final manuscript.

\section{Authors' information}

Jiuyang Liu and Chaoqun Huang are considered as co-first authors.

\section{Ethics approval and consent to participate}

Written informed consent was obtained from the patients with the study protocol approved by the ethics committee of Zhongnan Hospital of Wuhan University. The study was undertaken in accordance with the ethical standards of the World Medical Association Declaration of Helsinki.

\section{Consent for publication}

Informed consent to publish was obtained from each patient that was recruited.

\section{Competing interests}

The authors declare that they have no competing interests.

\section{Publisher's Note}

Springer Nature remains neutral with regard to jurisdictional claims in published maps and institutional affiliations.

\section{Author details}

${ }^{1}$ Department of Gastrointestinal Surgery, Zhongnan Hospital of Wuhan University, No. 169 Donghu Road, Wuchang District, Wuhan, China. ${ }^{2}$ Hubei Key Laboratory of Tumor Biological Behaviors \& Hubei Cancer Clinical Study Center, Wuhan 430071, China. ${ }^{3}$ Department of Thyroid and Breast Surgery, Zhongnan Hospital of Wuhan University, Wuhan, China. ${ }^{4}$ Department of General Surgery, Yingshan Renmin Hospital, Yingshan 438700, China. ${ }^{5}$ Department of Peritoneal Cancer Surgery, Beijing Shijitan Hospital, Capital Medical University, Beijing 100038, China. ${ }^{6}$ Peritoneal Dissemination Center, 
Kishiwada Tokushukai Hospital, Kishiwada 596-0032, Japan. ${ }^{7}$ Department of Surgery, Kusatsu General Hospital, Shiga 600-8189, Japan.

Received: 23 May 2018 Accepted: 1 November 2018 Published online: 12 November 2018

\section{References}

1. Ferlay J, Soerjomataram I, Dikshit R, Eser S, Mathers C, Rebelo M, Parkin DM, Forman D, Bray F. Cancer incidence and mortality worldwide: sources, methods and major patterns in GLOBOCAN 2012. Int J Cancer. 2015;136: E359-86.

2. Ferro A, Peleteiro B, Malvezzi M, Bosetti C, Bertuccio P, Levi F, Negri E, La Vecchia C, Lunet N. Worldwide trends in gastric cancer mortality (19802011), with predictions to 2015, and incidence by subtype. Eur J Cancer. 2014:50:1330-44.

3. Liu J, Geng X, Li Y. Milky spots: omental functional units and hotbeds for peritoneal cancer metastasis. Tumour Biol. 2016:37:5715-26.

4. Yonemura Y, Endou Y, Shinbo M, Sasaki T, Hirano M, Mizumoto A, Matsuda T, Takao N, Ichinose M, Mizuno M, Miura M, Ikeda M, Ikeda S, Nakajima G, Yonemura J, Yuuba T, Masuda S, Kimura H, Matsuki N. Safety and efficacy of bidirectional chemotherapy for treatment of patients with peritoneal dissemination from gastric cancer: selection for cytoreductive surgery. J Surg Oncol. 2009;100:311-6.

5. Glockzin G, Piso P. Current status and future directions in gastric cancer with peritoneal dissemination. Surg Oncol Clin N Am. 2012;21:625-33.

6. Huang CQ, Yang XJ, Yu Y, Wu HT, Liu Y, Yonemura Y, Li Y. Cytoreductive surgery plus hyperthermic intraperitoneal chemotherapy improves survival for patients with peritoneal carcinomatosis from colorectal cancer: a phase II study from a Chinese center. PLoS One. 2014;9:e108509.

7. Turley SJ, Cremasco V, Astarita JL. Immunological hallmarks of stromal cells in the tumour microenvironment. Nat Rev Immunol. 2015;15:669-82.

8. Zhan HX, Zhou B, Cheng YG, Xu JW, Wang L, Zhang GY, Hu SY. Crosstalk between stromal cells and cancer cells in pancreatic cancer: new insights into stromal biology. Cancer Lett. 2017;392:83-93.

9. Hanahan D, Weinberg RA. Hallmarks of cancer: the next generation. Cell. 2011;144:646-74.

10. Erdogan B, Ao M, White LM, Means AL, Brewer BM, Yang L, Washington MK, Shi C, Franco OE, Weaver AM, Hayward SW, Li D, Webb DJ. Cancer-associated fibroblasts promote directional cancer cell migration by aligning fibronectin. J Cell Biol. 2017;216:3799-816.

11. Zhang Q, Peng C. Cancer-associated fibroblasts regulate the biological behavior of cancer cells and stroma in gastric cancer. Oncol Lett. 2018;15:691-8.

12. Chong $Y$, Tang $D$, Xiong $Q$, Jiang $X, X u C$, Huang $Y$, Wang J, Zhou H, Shi $Y$, Wu X, Wang D. Galectin-1 from cancer-associated fibroblasts induces epithelial-mesenchymal transition through $\beta 1$ integrin-mediated upregulation of Gli1 in gastric cancer. J Exp Clin Cancer Res. 2016;35(1):175.

13. Mikuła-Pietrasik J, Uruski P, Tykarski A, Książek K. The peritoneal "soil" for a cancerous "seed": a comprehensive review of the pathogenesis of intraperitoneal cancer metastases. Cell Mol Life Sci. 2018;75:509-25.

14. Yang $X$, Lin Y, Shi Y, Li B, Liu W, Yin W, Dang Y, Chu Y, Fan J, He R. FAP promotes immunosuppression by cancer-associated fibroblasts in the tumor microenvironment via STAT3-CCL2 signaling. Cancer Res. 2016;76:4124-35.

15. Liao Y, Ni Y, He R, Liu W, Du J. Clinical implications of fibroblast activation protein-a in non-small cell lung cancer after curative resection: a new predictor for prognosis. J Cancer Res Clin Oncol. 2013;139:1523-8.

16. Patsouras D, Papaxoinis K, Kostakis A, Safioleas MC, Lazaris AC, Nicolopoulou-Stamati P. Fibroblast activation protein and its prognostic significance in correlation with vascular endothelial growth factor in pancreatic adenocarcinoma. Mol Med Rep. 2015;11:4585-90.

17. Hu M, Qian C, Hu Z, Fei B, Zhou H. Biomarkers in tumor microenvironment? Upregulation of fibroblast activation protein-a correlates with gastric cancer progression and poor prognosis. OMICS. 2017;21:38-44.

18. Wu H, Liu S, Gong J, Liu J, Zhang Q, Leng X, Zhang N, Li Y. VCPA, a novel synthetic derivative of a-tocopheryl succinate, sensitizes human gastric cancer to doxorubicin-induced apoptosis via ROS-dependent mitochondrial dysfunction. Cancer Lett. 2017;393:22-32.

19. Henry LR, Lee HO, Lee JS, Klein-Szanto A, Watts P, Ross EA, Chen WT, Cheng JD. Clinical implications of fibroblast activation protein in patients with colon cancer. Clin Cancer Res. 2007;13:1736-41.
20. Cohen SJ, Alpaugh RK, Palazzo I, Meropol NJ, Rogatko A, Xu Z, Hoffman JP, Weiner LM, Cheng JD. Fibroblast activation protein and its relationship to clinical outcome in pancreatic adenocarcinoma. Pancreas. 2008;37:154-8.

21. Goscinski MA, Suo Z, Florenes VA, Vlatkovic L, Nesland JM, Giercksky KE. FAP-alpha and UPA show different expression patterns in premalignant and malignant esophageal lesions. Ultrastruct Pathol. 2008;32:89-96.

22. Chen H, Yang WW, Wen QT, Xu L, Chen M. TGF-beta induces fibroblast activation protein expression; fibroblast activation protein expression increases the proliferation, adhesion, and migration of HO-8910PM [corrected]. Exp Mol Pathol. 2009;87:189-94.

23. Liu JY, Peng CW, Yang GF, Hu WQ, Yang XJ, Huang CQ, Xiong B, Li Y. Distribution pattern of tumor associated macrophages predicts the prognosis of gastric cancer. Oncotarget. 2017;8:92757-69.

24. Peng C, Liu J, Yang G, Li Y. Lysyl oxidase activates cancer stromal cells and promotes gastric cancer progression: quantum dot-based identification of biomarkers in cancer stromal cells. Int J Nanomedicine. 2017;13:161-74.

25. Peng C, Liu J, Yang G, Li Y. The tumor-stromal ratio as a strong prognosticator for advanced gastric cancer patients: proposal of a new TSNM staging system. J Gastroenterol. 2018;53:606-17.

26. Li P, Shan JX, Chen XH, Zhang D, Su LP, Huang XY, Yu BQ, Zhi QM, Li CL, Wang YQ, Tomei S, Cai Q, Ji J, Li JF, Chouchane L, Yu YY, Sun FZ, Xu ZH, Liu BY, Zhu ZG. Epigenetic silencing of microRNA-149 in cancer-associated fibroblasts mediates prostaglandin E2/interleukin-6 signaling in the tumor microenvironment. Cell Res. 2015;25:588-603.

27. Hanahan D, Weinberg RA. The hallmarks of cancer. Cell. 2000;100:57-70.

28. Fang M, Yuan J, Peng C, Li Y. Collagen as a double-edged sword in tumor progression. Tumour Biol. 2014;35:2871-82.

29. Li Z, Jo J, Jia JM, Lo SC, Whitcomb DJ, Jiao S, Cho K, Sheng M. Caspase-3 activation via mitochondria is required for long-term depression and AMPA receptor internalization. Cell. 2010;141:859-71.

30. Li X, Xie H, Chen Y, Lang M, Chen Y, Shi L. Silkworm Pupa Protein Hydrolysate Induces Mitochondria-Dependent Apoptosis and S PhaseCell Cycle Arrest in Human Gastric Cancer SGC-7901 Cells. Int J Mol Sci. 2018;19: E1013.

31. Sun Y, Miao H, Ma S, Zhang L, You C, Tang F, Yang C, Tian X, Wang F, Luo Y, Lin X, Wang H, Li C, Li Z, Yu H, Liu X, Xiao Y, Gong Y, Zhang J, Quan H, Xie C. FePt-Cys nanoparticles induce ROS-dependent cell toxicity, and enhance chemo-radiation sensitivity of NSCLC cells in vivo and in vitro. Cancer Lett. 2018;418:27-40.

32. Tan TZ, Miow QH, Miki Y, Noda T, Mori S, Huang RY, Thiery JP. Epithelialmesenchymal transition spectrum quantification and its efficacy in deciphering survival and drug responses of cancer patients. EMBO Mol Med. 2014;6:1279-93.

33. Vu T, Datta PK. Regulation of EMT in colorectal Cancer: a culprit in metastasis. Cancers (Basel). 2017;9:E171.

34. Stemmer V, de Craene B, Berx G, Behrens J. Snail promotes Wnt target gene expression and interacts with beta-catenin. Oncogene. 2008;27:5075-80.

35. Qi L, Sun B, Liu Z, Cheng R, Li Y, Zhao X. Wnt3a expression is associated with epithelial-mesenchymal transition and promotes colon cancer progression. J Exp Clin Cancer Res. 2014;33:107.

36. Shou J, Ali-Osman F, Multani AS, Pathak S, Fedi P, Srivenugopal KS. Human Dkk-1, a gene encoding a Wnt antagonist, responds to DNA damage and its overexpression sensitizes brain tumor cells to apoptosis following alkylation damage of DNA. Oncogene. 2002;21:878-89.

\section{Ready to submit your research? Choose BMC and benefit from:}

- fast, convenient online submission

- thorough peer review by experienced researchers in your field

- rapid publication on acceptance

- support for research data, including large and complex data types

- gold Open Access which fosters wider collaboration and increased citations

- maximum visibility for your research: over $100 \mathrm{M}$ website views per year

At BMC, research is always in progress.

Learn more biomedcentral.com/submissions 